\title{
Separation Process of Nisin Using Aqueous Two-Phase Systems: A New Approach Featuring Nanoparticles
}

Niloofar Rahbari, ${ }^{\dagger}$ Shahla Shahriari, ${ }^{*},+$ Gholamreza Pazuki ${ }^{\S}$

†Department of Food Science and Technology, Shahr-e-Qods Branch, Islamic Azad University, Tehran 37541-98811, Iran

$¥$ Department of Chemical Engineering, Shahr-e-Qods Branch, Islamic Azad University, Tehran 37541-98811, Iran

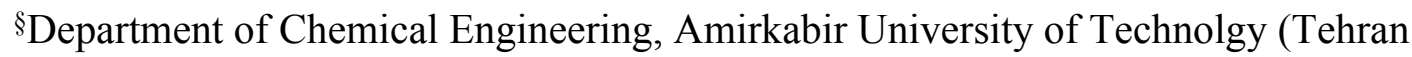

Polytechnic), Tehran 1591634311, Iran

- AUTHOR INFORMATION

Corresponding Author

*E-mail: shahla_shahriari@yahoo.com. Phone: +98 2146896000. 


\section{Supporting information}

Content:

- S1. Experimental mass fraction composition and partition coefficients of nisin in PEG $(1)+\mathrm{K}_{2} \mathrm{HPO}_{4}(2)+\mathrm{KH}_{2} \mathrm{PO}_{4}(3)+\mathrm{Nisin}(4)+\mathrm{H}_{2} \mathrm{O}(5)+$ nanoparticle $(6)+$ citric acid $(\approx$ $5.7 \%$ ) at $101 \mathrm{kPa}$ and $\mathrm{T}=298 \mathrm{~K}$. 


\section{Supporting information}

S1. Experimental mass fraction composition and partition coefficients of nisin in PEG (1) + $\mathrm{K}_{2} \mathrm{HPO}_{4}(2)+\mathrm{KH}_{2} \mathrm{PO}_{4}(3)+\mathrm{Nisin}(4)+\mathrm{H}_{2} \mathrm{O}(5)+$ nanoparticle $(6)+$ citric acid $(\approx 5.7 \%)$ at 101 $\mathrm{kPa}$ and $\mathrm{T}=298 \mathrm{~K}$.

\begin{tabular}{|c|c|c|c|c|c|c|c|c|c|c|}
\hline \multirow{3}{*}{ Nanoparticle } & \multicolumn{8}{|c|}{ mass fraction composition / wt $\%$} & \multirow{3}{*}{$\mathrm{K}$} & \multirow{3}{*}{$\mathrm{K}$ (average) $\pm \sigma^{2}$} \\
\hline & \multicolumn{6}{|c|}{ feed } & \multirow[b]{2}{*}{$\left(w_{4}\right)_{\text {top }}$} & \multirow[b]{2}{*}{$\left(\mathrm{w}_{4}\right)_{\text {bottom }}$} & & \\
\hline & $\mathrm{w}_{1}$ & $\mathrm{w}_{2}$ & $\mathrm{w}_{3}$ & $\mathrm{w}_{4}$ & $\mathrm{w}_{5}$ & $\mathrm{w}_{6}$ & & & & \\
\hline \multicolumn{11}{|c|}{$\mathrm{Mw}(\mathrm{PEG})=4000$} \\
\hline \multirow{2}{*}{$\begin{array}{c}\text { without } \\
\text { nano }\end{array}$} & 9.08 & 7.95 & 5.70 & 0.11 & 71.39 & 0.00 & 1.49 & 1.42 & 1.05 & \multirow{2}{*}{$1.02 \pm 0.04$} \\
\hline & 9.23 & 8.04 & 5.75 & 0.11 & 71.40 & 0.00 & 1.50 & 1.51 & 1.00 & \\
\hline \multirow{2}{*}{$\mathrm{n}-\mathrm{Gr}$} & 8.97 & 7.86 & 5.63 & 0.12 & 71.76 & 0.01 & 0.93 & 0.38 & 2.44 & \multirow{2}{*}{$2.47 \pm 0.04$} \\
\hline & 8.75 & 7.65 & 5.45 & 0.12 & 72.47 & 0.01 & 0.90 & 0.36 & 2.50 & \\
\hline \multirow{2}{*}{$\mathrm{n}-\mathrm{Fe}_{2} \mathrm{O}_{3}$} & 8.97 & 7.86 & 5.63 & 0.12 & 71.76 & 0.01 & 0.61 & 4.07 & 0.15 & \multirow{2}{*}{$0.15 \pm 0.00$} \\
\hline & 8.97 & 7.86 & 5.63 & 0.12 & 71.76 & 0.01 & 0.65 & 4.01 & 0.16 & \\
\hline \multirow{2}{*}{$\mathrm{n}-\mathrm{ZnO}$} & 9.21 & 8.08 & 5.74 & 0.11 & 71.07 & 0.01 & 1.54 & 1.38 & 1.11 & \multirow{2}{*}{$1.08 \pm 0.04$} \\
\hline & 9.19 & 8.03 & 5.75 & 0.11 & 71.16 & 0.01 & 0.88 & 0.84 & 1.05 & \\
\hline \multirow{2}{*}{$\mathrm{n}-\mathrm{Ag}$} & 9.21 & 8.08 & 5.74 & 0.11 & 71.07 & 0.01 & 1.46 & 1.29 & 1.13 & \multirow{2}{*}{$1.14 \pm 0.02$} \\
\hline & 9.19 & 8.03 & 5.75 & 0.11 & 71.16 & 0.01 & 1.18 & 1.02 & 1.16 & \\
\hline \multirow{2}{*}{ Funct.n-Gr } & 8.97 & 7.86 & 5.63 & 0.12 & 71.76 & 0.01 & 5.14 & 1.47 & 3.49 & \multirow{2}{*}{$3.78 \pm 0.42$} \\
\hline & 8.75 & 7.69 & 5.45 & 0.12 & 72.47 & 0.01 & 5.81 & 1.42 & 4.09 & \\
\hline \multicolumn{11}{|c|}{$\mathrm{Mw}(\mathrm{PEG})=6000$} \\
\hline \multirow{2}{*}{$\begin{array}{c}\text { without } \\
\text { nano }\end{array}$} & 9.17 & 8.04 & 5.77 & 0.12 & 71.10 & 0.00 & 0.69 & 0.67 & 1.03 & \multirow{2}{*}{$1.04 \pm 0.00$} \\
\hline & 9.17 & 8.07 & 5.78 & 0.12 & 71.07 & 0.00 & 0.67 & 0.65 & 1.04 & \\
\hline & 8.97 & 7.86 & 5.63 & 0.12 & 71.76 & 0.01 & 1.01 & 0.32 & 3.20 & \\
\hline n-Gr & 8.75 & 7.65 & 5.45 & 0.12 & 72.47 & 0.01 & 1.11 & 0.39 & 2.88 & $3.04 \pm 0.22$ \\
\hline & 8.97 & 7.86 & 5.63 & 0.12 & 71.76 & 0.01 & 1.23 & 2.83 & 0.44 & \\
\hline $\mathrm{n}-\mathrm{Fe}_{2} \mathrm{O}_{3}$ & 8.97 & 7.86 & 5.63 & 0.12 & 71.76 & 0.01 & 1.30 & 2.25 & 0.58 & $0.51 \pm 0.09$ \\
\hline & 9.21 & 8.08 & 5.74 & 0.11 & 71.07 & 0.01 & 1.37 & 1.33 & 1.03 & $101+003$ \\
\hline $\mathrm{n}-\angle \mathrm{n} U$ & 9.19 & 8.03 & 5.75 & 0.11 & 71.16 & 0.01 & 1.36 & 1.37 & 0.99 & $1.01 \pm 0.03$ \\
\hline & 9.21 & 8.08 & 5.74 & 0.11 & 71.07 & 0.01 & 0.08 & 0.07 & 1.17 & $122+006$ \\
\hline$n-A g$ & 9.19 & 8.03 & 5.75 & 0.11 & 71.16 & 0.01 & 0.09 & 0.07 & 1.27 & $1.22 \pm 0.06$ \\
\hline & 9.19 & 8.02 & 5.78 & 0.12 & 71.11 & 0.01 & 6.31 & 1.41 & 4.48 & \\
\hline Funct.n-Gr & 9.17 & 8.04 & 5.76 & 0.13 & 71.13 & 0.01 & 5.91 & 1.41 & 4.20 & $4.34 \pm 0.19$ \\
\hline & & & & & Iw (PEC & $=10000$ & & & & \\
\hline without & 9.21 & 8.06 & 5.77 & 0.12 & 71.03 & 0.00 & 0.74 & 0.67 & 1.10 & $1.16+0.08$ \\
\hline nano & 9.08 & 7.94 & 5.67 & 0.11 & 71.47 & 0.00 & 0.73 & 0.60 & 1.22 & $1.16 \pm 0.08$ \\
\hline & 8.97 & 7.86 & 5.63 & 0.12 & 71.76 & 0.01 & 1.59 & 0.34 & 4.64 & $414+069$ \\
\hline n-Gr & 8.75 & 7.65 & 5.45 & 0.12 & 72.47 & 0.01 & 1.55 & 0.42 & 3.65 & $4.14 \pm 0.69$ \\
\hline & 8.97 & 7.86 & 5.63 & 0.12 & 71.76 & 0.01 & 2.79 & 1.69 & 1.65 & $121+061$ \\
\hline$n-\mathrm{Fe}_{2} \mathrm{O}_{3}$ & 8.97 & 7.86 & 5.63 & 0.12 & 71.76 & 0.01 & 1.07 & 1.37 & 0.78 & $1.21 \pm 0.61$ \\
\hline$n-7 n O$ & 9.21 & 8.08 & 5.74 & 0.11 & 71.07 & 0.01 & 1.72 & 1.43 & 1.20 & $116+005$ \\
\hline $\mathrm{n}-\mathrm{ZnO}$ & 9.19 & 8.03 & 5.75 & 0.11 & 71.16 & 0.01 & 1.53 & 1.36 & 1.13 & $1.16 \pm 0.05$ \\
\hline & 9.21 & 8.08 & 5.74 & 0.11 & 71.07 & 0.01 & 0.25 & 0.17 & 1.49 & $153+0.06$ \\
\hline n-Ag & 9.19 & 8.03 & 5.75 & 0.11 & 71.16 & 0.01 & 0.27 & 0.17 & 1.57 & $1.53 \pm 0.06$ \\
\hline Funct $n$ & 9.21 & 8.08 & 5.74 & 0.11 & 71.07 & 0.01 & 5.04 & 0.67 & 7.53 & $817+0.97$ \\
\hline Funct.n-Gr & 9.19 & 8.03 & 5.75 & 0.11 & 71.16 & 0.01 & 5.82 & 0.66 & 8.83 & $8.1 / \pm 0.92$ \\
\hline
\end{tabular}

a Standard deviation. Standard uncertainties: $\mathrm{u}(\mathrm{T})=0.5 \mathrm{~K} ; \mathrm{u}(\mathrm{P})=10 \mathrm{kPa}$. 